\title{
Special Issue "Observations of Coupled Processes in Fractured Geological Media at Various Space and Time"
}

\author{
Ki-Bok Min ${ }^{1} \cdot$ Jonny Rutqvist ${ }^{2} \cdot$ Victor Vilarrasa $^{3}$
}

Accepted: 14 September 2020 / Published online: 6 October 2020

(c) Springer-Verlag GmbH Austria, part of Springer Nature 2020

\section{Introduction}

This Special Issue originates from selected contributions at the International Conference on Coupled Processes in Fractured Geological Media: Observation, Modeling, and Application (CouFrac2018). CouFrac2018 has been the first CouFrac conference focusing on coupled processes in fractured media, providing a platform for international discussion, communication and networking on this emerging topic. The conference was held in Wuhan, China, between 12 and 14th of November, 2018.

CouFrac2018 was co-organized by the Lawrence Berkeley National Laboratory, Seoul National University and the Institute of Rock and Soil Mechanics, Chinese Academy of Science. An organizing committee of 37 members contributed to a successful organization of the conference.

CouFrac2018 was attended by 170 participants from 17 countries. There was a panel discussion, a Distinguished Invited Talk by Nick Barton, 7 Keynote Presentations and a Chin-Fu Tsang Coupled Processes Award Lecture by Adriana Paluszny of Imperial College. The scientific contributions were presented in 24 technical sessions, including 96 talks, 11 of which were invited talks, and 42 posters.

This special issue in Rock Mechanics and Rock Engineering is focused on laboratory and in situ observations of coupled process in fractured rock with ten selected papers, all of which have been peer reviewed. The special issue intents to present the state of the art in coupled

Ki-Bok Min

kbmin@snu.ac.kr

1 Department of Energy Resources Engineering, Seoul National University (SNU), Seoul, South Korea

2 Energy Geosciences Division, Lawrence Berkeley National Laboratory (LBNL), Berkeley, USA

3 Institute of Environmental Assessment and Water Research (IDAEA), Spanish National Research Council (CSIC), Madrid, Spain thermal-hydro-mechanical-chemical (THMC) processes in fractured geological media, specifically focusing on observations ranging from microscale (micron to centimeter) to macroscale (meter to kilometer) and at multiple time scales (from nanoseconds to thousands of years). This includes various studies from laboratories, underground research laboratories, in situ demonstration and field observations often in combination with numerical modeling unveiling key physical processes.

While proper understanding on the interrelated phenomena of hydraulic, thermal, mechanical and chemical process occurring in fractured geological media is essential for numerous applications, our understanding of many aspects of such coupled processes are not fully understood. The ten papers compiled in this special issues clearly demonstrates active research being carried out around the world. We would like to extend special gratitude to the authors of the ten papers for their contributions.

\section{The Special Issue}

The Special Issue covers recent developments in the understanding of permeability evolution of fractured rock during hydro-shearing (Kakurina et al. 2020; Shen et al. 2020), fracturing mechanisms of fractured rock (Zhuang et al. 2020; Pan et al. 2020; Li et al. 2020; Zhou et al. 2020), effect of high and low temperature on various rock properties (Yang et al. 2020; Weng et al. 2020) and characterization of transport and mechanical characteristics of rock under different conditions (Suzuki et al. 2020; Zhang et al. 2020).

Kakurina et al. (2020) investigate the orientation of injection-induced fault reactivation in two in situ experiments, one performed in carbonate rock at the Rustrel Low Noise Underground Laboratory (LSBB URL), France, and another in shale rock at the Mont Terri Rock laboratory, Switzerland. In both cases, pre-exiting fractures deform aseismically, leading to irreversible shear slip and opening of the 
fractures. The authors develop a methodology to calculate slip vectors from displacement rates during two phases. A first set of slip vectors can be used to estimate the background stress state, whereas deviations from the first slip vectors provide information about fracture reactivation. The distinct two phases identified in the current study from the underground research laboratories have potential to be further used in various applications requiring fluid injection.

Shen et al. (2020) investigate the dynamic permeability evolution in the direction parallel with shear slip of six rough granite fractures under different effective normal stress. As shear slip accumulates, permeability first increases due to dilatancy, but subsequently decreases at effective normal stress lower than $5 \mathrm{MPa}$. At higher effective normal stress, fault gouge formation leads to a continuous permeability reduction with shear slip. The study shows that the characterization of fracture permeability increase during shear is not straightforward and extensive research is necessary in terms of the role of normal stress, roughness and formation of fault gouge.

Zhuang et al. (2020) examine the injectivity enhancement and acoustic emissions when creating hydraulic fractures in cubic granite samples under six fluid injection schemes in true triaxial experiments. The stepwise pulse pressurization scheme creates the largest hydraulic fracture, providing the highest permeability increase. While the cyclic progressive injection induces the lowest amplitude of the acoustic emissions, it yields the lowest permeability increase. The cyclic pulse pressurization provides a balance between a reasonable increase in permeability and relatively limited induced seismicity. All injection schemes generate hydraulic fractures that, at the grain scale forms intragranular fractures splitting microcline, orthoclase and quartz. The laboratory test results from this study show the potential for optimized hydraulic stimulation while minimizing the risk on induced seismicity in the field applications by advanced fluid injection schemes.

Pan et al. (2020) investigate the effect of flaw inclination and infilling on the flaw surface relative displacement and on the induced cracking behavior. The authors perform uniaxial compression experiments in flawed marble varying the flaw inclination from horizontal to vertical in open flaw and flaw filled with gypsum, cement and resin. Unfilled flaws give rise to the largest relative displacements. The stiffer the filler of flaws in hard rock, the wing cracks initiate closer to the flaw tips and with a lower angle, increasing the likelihood of quasi-coplanar crack initiation and suppressing the initiation of anti-wing cracks from the flaw tips. This fundamental study on the fracturing behavior of flawed hard rock under mechanical loading laid the foundation for several applications, including hydraulic fracturing.

$\mathrm{Li}$ et al. (2020) use gels to improve the understanding of hydraulic fracturing initiation and propagation. The experimental observations fit the theoretical estimates quite well, but discrepancies increase as the fracture grows. The experimental results are scaled up to the field scale assuming a tight rock, predicting fracture radius in the order of tens of meters. While a more accurate correction is needed for vertical fractures evolving beyond the formation thickness, the study demonstrated that gelatin block can be used as a material to simulate hydraulic fracturing.

Zhou et al. (2020) propose a novel numerical method that combines strength and energy to simulate hydraulic fracture propagation in the presence of natural fractures. This method, which has been implemented in an extended finite element method (XFEM) scheme, permits simulating the interaction between the hydraulic fracture and pre-existing natural fractures, reproducing the crossing behavior when a natural fracture slips and/or opens as a result of the hydraulic fracture terminating into it. Arrest, crossing and offset of hydraulic fractures with pre-existing natural fractures anticipated in the field were reproduced in the numerical study.

Yang et al. (2020) study the permeability evolution of granite at very high temperatures (up to $800{ }^{\circ} \mathrm{C}$ ) to reproduce conditions various applications including nuclear waste disposal. A permeability increase of four orders of magnitude is measured in intact granite samples for a temperature increase from 25 to $800^{\circ} \mathrm{C}$. While thermally induced cracks control failure at temperatures above $450{ }^{\circ} \mathrm{C}$ under uniaxial compression conditions, temperature has little effect on failure under triaxial compression conditions, which forms a shear fracture plane. The presence of thermally induced cracks becomes significant at temperatures above $300{ }^{\circ} \mathrm{C}$, reducing the strength of the rock. A variety of measurements with temperatures up to $800{ }^{\circ} \mathrm{C}$ offer a useful dataset for further studies.

Weng et al. (2020) measure dynamic mechanical properties of dry and water-saturated siltstones at sub-zero temperatures to gain knowledge on the response of this rock for geotechnical applications affected by extreme cold. Both the dynamic uniaxial compressive strength and the dynamic Young's modulus increase as the temperature decreases from 18 to $-30{ }^{\circ} \mathrm{C}$, but the modulus subsequently decrease when temperatures decrease down to $-50{ }^{\circ} \mathrm{C}$. Furthermore, saturated samples compact faster than dry samples at sub-zero temperatures. Despite the sub-zero temperature, only a portion of the pore water freezes, reaching an ice content close to $90 \%$ at $-50{ }^{\circ} \mathrm{C}$. Several phenomena affect the evolution of the mechanical properties with temperature, including the shrinkage of mineral grains, the enhancement of the ice strength and the interaction of the water/ice mixture with rock as the temperature drops.

Suzuki et al. (2020) perform flow experiments in a micromodel replicating a single fracture and the rock matrix to investigate transport of nano- and microparticles. By measuring the distribution of particle sizes at the outlet, 
the authors found that while the portion of small particles arrives in the outlet from early to late times, the portion of large particles arrives only at early times. This observation suggests that large particles travel only along the fracture, but small particles travel both along the fracture and through the rock matrix. Thus, the size of the injected particles in tracer tests can be used to characterize fracture structures, such as the critical aperture in flow pathways.

Zhang et al. (2020) investigate how salt precipitation affects porosity, permeability, permeability-stress sensitivity and elastic wave velocities of high-salinity tight sandstone, a relevant effect in natural gas extraction. Salt precipitation significantly reduce porosity, permeability and elastic wave velocities. Microstructural analysis reveals that crystalline salt precipitates in the pores, microfractures and on the surface of clay minerals, limiting the flow pathways to the smaller pores and pore throats and inducing secondary cracks that weaken the rock. The study is a valuable addition to the scarce data related to the influence of salt precipitation on the properties of a tight sandstone gas reservoirs.

Acknowledgements K.-B.M. acknowledges support from the Institute of Engineering Research, Seoul National University. J.R. acknowledges support from the U.S. Department of Energy to the Lawrence Berkeley National Laboratory under contract No. DE-AC02-05CH11231. V.V. acknowledges funding from the European Research Council (ERC) under the European Union's Horizon 2020 Research and Innovation Programme through the Starting Grant GEoREST, grant agreement No. 801809 (www.georest.eu).

\section{References}

Kakurina M, Guglielmi Y, Nussbaum C, Valley B (2020) In-situ direct displacement information on fault reactivation during fluid injection. Rock Mech Rock Eng. https://doi.org/10.1007/s00603-02002160-w
Li Z, Wang J, Gates ID (2020) Fracturing gels as analogs to understand fracture behavior in shale gas reservoirs. Rock Mech Rock Eng. https://doi.org/10.1007/s00603-020-02153-9

Pan PZ, Miao S, Jiang Q, Wu Z, Shao C (2020) The influence of infilling conditions on flaw surface relative displacement induced cracking behavior in hard rock. RockMech Rock Eng. https://doi. org/10.1007/s00603-019-02033-x

Shen H, Zhang Q, Li Q, Li X, Shi L, Shen N (2020) Experimental and numerical investigations of the dynamic permeability evolution of a fracture in granite during shearing under different normal stress conditions. Rock Mech Rock Eng. https://doi.org/10.1007/ s00603-020-02074-7

Suzuki A, Cui J, Zhang Y, Uehara S, Li K, Horne RN, Ito T (2020) Experimental study on nano-/microparticles transport to characterize structures in fractured porous media. Rock Mech Rock Eng. https://doi.org/10.1007/s00603-020-02081-8

Weng L, Wu Z, Liu Q (2020) Dynamic mechanical properties of dry and water-saturated siltstones under sub-zero temperatures. Rock Mech Rock Eng. https://doi.org/10.1007/s00603-019-02039-5

Yang SQ, Tian WL, Elsworth D, Wang JG, Fan LF (2020) An experimental study of effect of high temperature on the permeability evolution and failure response of granite under triaxial compression. Rock Mech Rock Eng. https://doi.org/10.1007/s00603-01901982-7

Zhang D, Kang Y, Selvadurai APS, You L (2020) Experimental investigation of the effect of salt precipitation on the physical and mechanical properties of a tight sandstone. Rock Mech Rock Eng. https://doi.org/10.1007/s00603-019-02032-y

Zhou Y, Yang D, Zhang X, Chen W, Xiong Q (2020) A numerical method for fracture crossing based on average stress levels. Rock Mech Rock Eng. https://doi.org/10.1007/s00603-020-02054-X

Zhuang L, Jung SG, Diaz M, Kim KY, Hofmann H, Min KB, Zang A, Stephansson O, Zimmermann G, Yoon JS (2020) Laboratory true triaxial hydraulic fracturing of granite under six fluid injection schemes and grain-scale fracture observations. Rock Mech Rock Eng. https://doi.org/10.1007/s00603-020-02170-8

Publisher's Note Springer Nature remains neutral with regard to jurisdictional claims in published maps and institutional affiliations. 